\title{
A SINGLE-CAMERA SYNTHETIC SCHLIEREN METHOD FOR MEASURING TWO-DIMENSIONAL LIQUID SURFACES
}

\author{
Duo $\mathrm{Xu}^{*}$ \\ The State Key Laboratory of Nonlinear Mechanics \\ Institute of Mechanics, Chinese Academy of Sciences \\ Beijing, 100190, China \\ Email: duo.xu@imech.ac.cn \\ Center of Applied Space Technology and Microgravity \\ University of Bremen \\ Bremen, 28359, Germany
}

\author{
Huixin Li \\ Center of Applied Space Technology and Microgravity \\ University of Bremen \\ Bremen, 28359, Germany \\ Marc Avila \\ Center of Applied Space Technology and Microgravity \\ University of Bremen \\ Bremen, 28359, Germany \\ MAPEX Center for Materials and Processes \\ University of Bremen \\ Bremen, 28359, Germany
}

\begin{abstract}
A single-camera synthetic Schlieren method is introduced here to measure two-dimensional topography and depth of $d y$ namic free liquid surfaces. The method is simple and easy to implement. Because of light refraction (following Snell's law), markers on a flat bottom which are seen through the surfaces of a transparent liquid are virtually displaced. This leads to a governing equation that the liquid surface depth (and its topography) is associated with the marker displacement. In the equation, the refractive index of the liquid (e.g. water) can be obtained by a refractometer (or from a technical reference), and the displacements of the markers can be obtained by a cross-correlation method which is usually used in particle image velocimetry. In the equation, the only unknown, the depth of the surface, can be obtained by solving the governing equation with boundary conditions. Unlike free-surface synthetic Schlieren (FS-SS) of Moisy et al. (Exp. Fluids, 1021, 46, 2009), our method does not require a reference depth (which is obtained before or after experiments), so that flows with temporally evolving depth can be measured. Experiments of liquid ripples and dam-break flows
\end{abstract}

*Address all correspondence to this author. were performed to test the method. The results agree well with those obtained with FS-SS and visualization measurements.

\section{INTRODUCTION}

The measurement of a liquid free surface is fundamental and important in engineering applications, e.g. chemical, marine and coastal engineering, where hydrodynamics of liquid surface dominates the underlying physics. The liquid surface depth and slopes can be measured by a few methodologies, e.g. acoustic techniques and depth gauges, whereas optical techniques, e.g. imaging methods [1], are preferred due to the nature of nonintrusiveness

Jähne et al. [2] used a complex telecentric optical system (where image magnification does not change with the surface height) for measuring slopes/depths of the surface, which is calibrated to be correlated to the different light absorption rates from LEDs with red and near-infrared lights. Recently, Aureli et al. [3] developed the light-absorption-based imaging method in a camera with two CCDs (where one is a RGB Bayer array and another is a monochrome sensor). Note that precise alignments of colli- 
mated beams in different wavelengths are necessarily required for these methods.

A few variety of imaging techniques are based on the working principles of light reflection. The slope of two-dimensional surface viewed by an imaging polarimeter can be measured with the relationship between the surface orientation and the polarization change of the light reflected at the surface [4]. This technique can also be applied to unpolarized skylight for rivers. In order to enhance the light reflection, additives were added to water, while symbol patterns [5] and fringe patterns [6,7] were projected to the solution surface.

Other imaging techniques reply on the light refraction. In a channel of shallow water, Kurata et al. [8] used a camera to capture a reference image of a striped grating, which was placed below the channel, when the water was still. When surface ripples were produced, they took a refracted image of the grating, where the featured points were virtually displaced due to the light refraction on the water surface. Calculation of the point displacement gives the topographic structures of the surface, and the surface depth can be reconstructed if the depth of a reference point was known. Following the same working principle, Moisy et al. [1], Fouras et al. [9] and $\mathrm{Ng}$ et al. [10] used the random dot pattern by taking the advantage of optimized cross-correlation algorithm of particle image velocimetry (PIV) for extraction of the displacements in high spatial resolution. In the free-surface synthetic Schlieren (FS-SS) method of Moisy et al. [1], the ordinary derivative equation of the surface depth, which is derived from the Snell's law of the light refraction, was solved using leastsquare inversion of a linear system of the equations to obtain the surface topography. In order to reconstruct the surface depth, the spatially averaged height of the surface is needed and it must be approximately constant in time. Differing to the grating and random dot patterns, Zhang and Cox [11] introduced a color-coded pattern, which was carefully designed to correlate with different surface slopes one-to-one [12].

These refraction methods measure the surface topography using low-cost illumination, simple optical configuration and a single camera. However, to reconstruct the surface depth from the topographic structures, they need height references, which is either time-dependent or unknown in some flows. Morris [13] and Gomit et al. [14] used two or more cameras to establish stereo views of the same pattern. The measurements from the multiple views can directly determine the topography and the depth of the surface, e.g. by matching the surface orientation from the multiple views [13], without the reference height. The usage of multi-camera system increases the hardware costs and requires careful calibration of camera positions, while the postprocessing requires more computation power.

In this work, we introduce a synthetic Schlieren method to directly measure the depth of surface (and its topography) using a single camera, without neither any reference heights nor parametric models. This paper is organized as follows: The

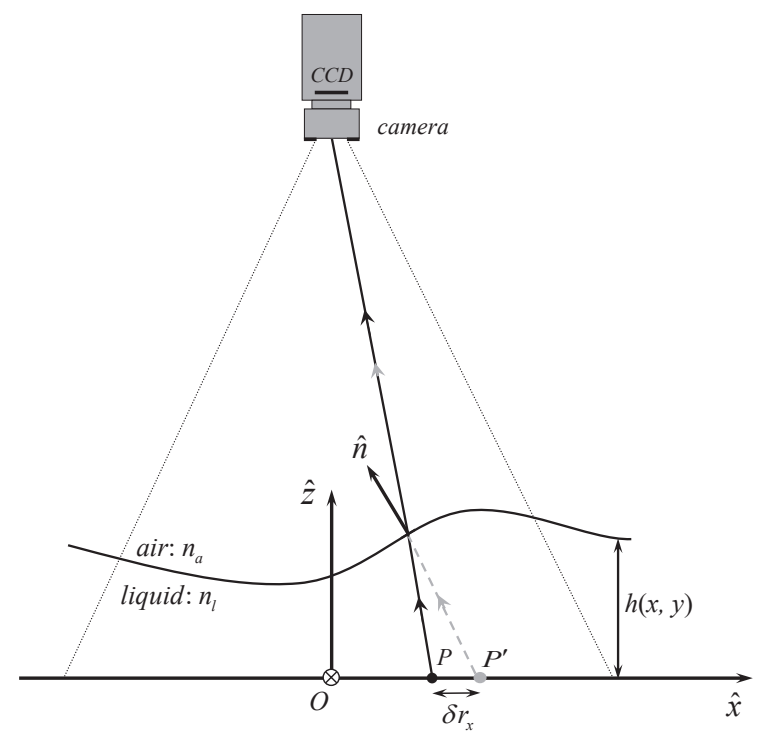

FIGURE 1. SCHEMATIC OF WORKING PRINCIPLE OF MEASURING THE TOPOGRAPHIC DEPTH OF A LIQUID INTERFACE.

working principle is introduced in Section METHODOLOGY. The experiments for examining the present method are given in Section EXPERIMENTS, where in experiments of liquid ripples spatially averaged depth is approximately constant, and in experiments of dam-break flows the averaged depth changes with time. The detailed discussion is given in Section LIMITATIONS AND DISCUSSION, and the conclusion is drawn in Section CONCLUSION.

\section{METHODOLOGY Working Principle}

The presented methodology is based on the refraction of light rays, resulted from different refractive indices of two fluids, at the air-liquid interface. This physical principle shares that of synthetic Schlieren method [1] and background-oriented Schlieren method [15].

A schematic that depicts the working principle is shown in Fig. 1. For a given point $P$, when there is no liquid, the incident light ray follows a straight black trajectory. When there is transparent liquid forming a liquid-air interface, the point $P$ shifts to $P^{\prime}$ due to the ray refraction at the interface, where the normal direction is $\widehat{\mathbf{n}}$. The light ray refraction is governed by the Snell's law for refractive index of the liquid $n_{l}$ and that of the air $n_{a}$. The $P-P^{\prime}$ displacement $\delta \mathbf{r}$ is produced by the liquid height $h(x, y)$, the surface topography $\nabla h(x, y)$ and the ratio of refractive indices 
$n_{a} / n_{l}$ specifically,

$$
\begin{aligned}
\delta \mathbf{r}= & -h \cdot \tan \left(\tan ^{-1}(\nabla h)+\sin ^{-1}\left[\left(n_{a} / n_{l}\right) \cdot \sin \left(\boldsymbol{\beta}-\tan ^{-1}(\nabla h)\right)\right]\right) \\
& +h \cdot \tan \boldsymbol{\beta} .
\end{aligned}
$$

Here $n_{a} / n_{l}$ is known from measurements of a refractometer, and $\delta \mathbf{r}(x, y)$ can be obtained by cross-correlation of the calibration and the refraction images (see Fig. 2b, c), and $\delta \mathbf{r}(x, y)$ can reach subpixel level of accuracy with a Gaussian peak-fit estimator [18]. The camera viewing angle, $\boldsymbol{\beta}(x, y)=\beta_{x}(x, y) \hat{x}+\beta_{y}(x, y) \hat{y}$, is determined by the spatial positions of the camera and the object pattern (e.g. see Fig. 2a), while $h(x, y)$ is the only unknown to be determined.

When the camera is set to satisfy the paraxial approximation and liquid surface is of weak slope, according to the small-angle approximation, the equation (1) can be simplified to

$$
\delta \mathbf{r}=-h \cdot\left(1-n_{a} / n_{l}\right) \nabla h+h \cdot\left(1-n_{a} / n_{l}\right) \boldsymbol{\beta} .
$$

The small-angle approximation is also used in the FS-SS method [1], where the spatial fluctuations of the liquid surface is assumed to be much smaller than the averaged depth, and the equation (2) is reduced to

$$
\delta \mathbf{r}^{\prime}=-h^{*} \cdot \nabla h^{\prime}
$$

where $h^{*} \approx \alpha h_{p}, \alpha$ is a constant given by the camera system (e.g. $\alpha \approx 0.24$ in [1]) and $h_{p}$ is the reference height, which is approximately the averaged depth of the surface, while $\delta \mathbf{r}^{\prime}(x, y)$ are the displacements between the pattern images from the flat surface in still and the fluctuated surface. This simplification enables $h^{\prime}(x, y)$ in the over-determined linear system of equation (3) to be solved by a least-square approach (e.g. the backslash operator in Matlab), and the reconstruction gives $h(x, y)=h^{\prime}(x, y)+h_{p}$ [1]. When the liquid fluctuation is strong, i.e. large surface slopes and large wave amplitudes, the imaged dots may be largely stretched, and the displacement accuracy may be decreased. This can be compensated by dot-tracking algorithms [16].

To measure the complex topographic liquid surface in high spatial resolution, numerous dots are used as reference objects (see Fig. 2b). In analogy to PIV, random dot markers are printed on a transparent sheet, which is attached on a flat bottom. The random dot pattern is numerically generated by a Matlab script [17]. The randomly distributed dots contributes to PIV correlation algorithm to reduce fortuitous pattern coincidence for better accuracy. We carefully design the pattern, where the diameters of individual dots are approximately 6 pixels in images.

In practice, $\boldsymbol{\beta}(x, y)$ can be determined by a simple calibration procedure: For a leveled flat bottom, still water (or a transparent solid block) with known $n_{l}$ gives a flat surface (i.e. $\nabla h=0$ ), while the liquid depth (or thickness of the solid) $h_{c}$ can be measured by a caliper precisely, so according to equation (2), $\boldsymbol{\beta}(x, y)$ can be obtained by

$$
\boldsymbol{\beta}(x, y)=\delta \mathbf{r}_{c}(x, y) /\left[h_{c}\left(1-n_{a} / n_{l}\right)\right],
$$

where $\delta \mathbf{r}_{c}(x, y)$ is obtained by the image correlation. See Fig. 2(a) for an example of $\boldsymbol{\beta}$.

\section{Surface Depth}

The displacements of the dot-pattern, $\delta \mathbf{r}(x, y)$, can be obtained by correlating the refraction images with the calibration image, and the latter is captured without liquid. Image crosscorrelation algorithms, as commonly used in two-dimensional PIV, are applied in this study. The multi-step algorithm with reduction of interrogation window $96 \times 96$ pixel $^{2}$ to the final window $24 \times 24$ pixel $^{2}$ with overlap of $75 \%$ is used in Lavision Davis. The final step interrogation window includes approximately 8 dots for accurate displacement tracking [18], given that the number of dots in each interrogation window between the paired images is approximately unchanged.

The displacements $\delta \mathbf{r}$ span over $M \times N$ grids, and the surface depth $h$ at the $M N$ grids are the unknowns to be solved. The unknown $h$ at each of $M N$ grids is governed by the equation (1) (or 2) along both (x and y) directions, and this leads to $2 M N$ equations in a linear system. A least-square algorithm based on the over-determined linear system is used in this study to obtain the converged solution of $h(x, y)$.

\section{Coordinate Remapping}

In Fig. 1, the displacement $\delta \mathbf{r}$ measured at the point $P$ originates from the intersection of a light ray with the wave surface. The coordinate $(x, y)$ of the intersection point differs from the point $P$, where the displacement coordinate is set. For the coordinate remapping, a method is used based on the fact that $h(x, y)$ and $\boldsymbol{\beta}(x, y)$ are solved and known, respectively, so that the remapped coordinates $\left(x^{*}, y^{*}\right) \approx(x, y)-h(x, y) \cdot \tan [\boldsymbol{\beta}(x, y)]$. The results in the following are plotted with the remapped coordinates, and note that $(x, y)$ is used as the remapped coordinates for simplicity.

\section{EXPERIMENTS}

In this section, we report the experiments of liquid ripples and dam-break problems, respectively. In the former case, spatially averaged surface depth is approximately constant, while the averaged depth in the latter temporally evolves. 

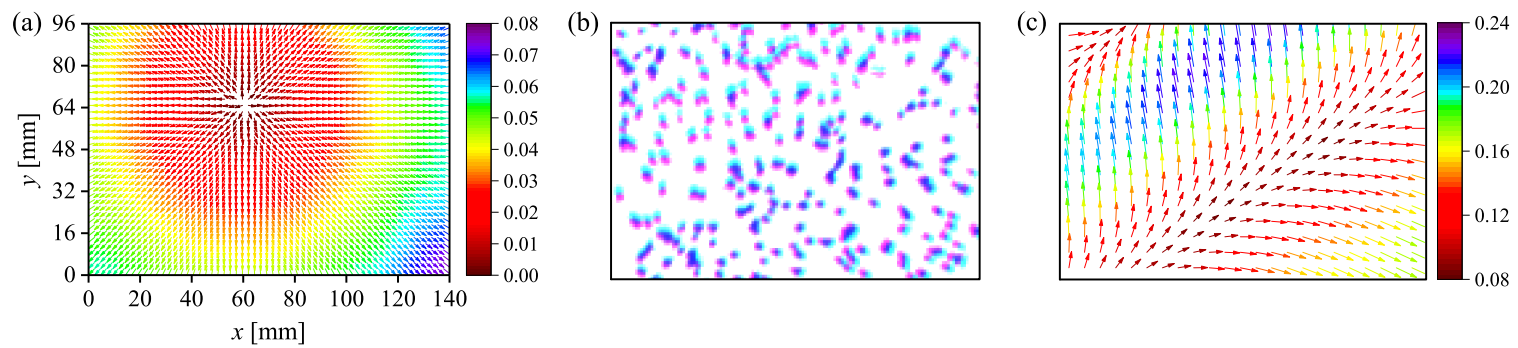

FIGURE 2. (a) THE VECTOR MAP OF $\boldsymbol{\beta}(x, y)=\left(\beta_{x}, \beta_{y}\right)$ OBTAINED IN THE CALIBRATION PROCEDURE, WHERE THE THE COLOR SHOWS THE MAGNITUDE OF $\boldsymbol{\beta}$. (b) A PORTION OF DOT PATTERN IS SHOWN: PINK DOTS (TUNED FROM BLACK) ARE FROM A CALIBRATION IMAGE, WHILE CYAN DOTS (TUNED FROM BLACK) ARE FROM A MEASUREMENT IMAGE OF SURFACE RIPPLES, AND THE OVERLAP IS IN BLUE. (c) THE DISPLACEMENTS $\delta$ r (AMPLIFIED 4 TIMES IN LENGTH FOR CLARITY) BETWEEN THE PINK AND THE CYAN DOTS IN (b).

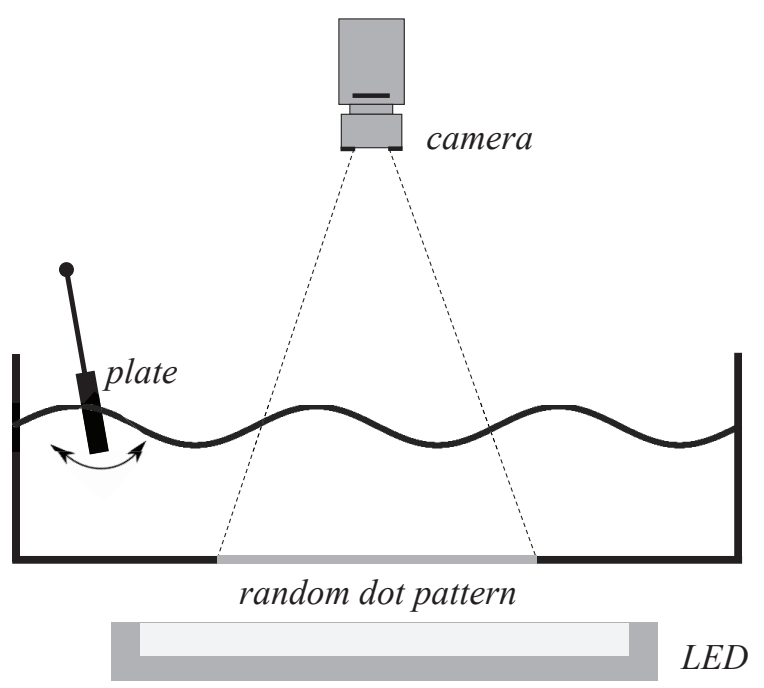

FIGURE 3. SCHEMATIC OF MEASURING TOPOGRAPHY AND DEPTH OF LIQUID RIPPLES IN A TANK.

\section{Liquid Ripples}

In this study, the liquid was water with refractive index $n_{l}=1.334$, and the air had $n_{a}=1$. The tank (in dimension of $1000 \times 150 \times 350 \mathrm{~mm}^{3}$ ) was manufactured by the transparent acrylic plates. The random dot pattern printed on an overhead transparency (with thickness of $0.16 \mathrm{~mm}$ ) in a printer (Ricoh MP C3504) had a dimension of $200 \mathrm{~mm} \times 120 \mathrm{~mm}$, and it was attached upon the flat bottom of the tank. A light-emitting diode (LED) illuminated the random dot pattern from the bottom of the tank, while a camera (Phantom VEO 640L equipped with a Zeiss lens of focal length $100 \mathrm{~mm}$ ) in resolution $2560 \times 1600$ pixel $^{2}$ was placed approximately 1 meter above the pattern. A plate powered by a motor was employed to flap recursively to generate liquid ripples (see Fig. 3).

The experimental procedure is as follows: First, without any water $\left(20^{\circ} \mathrm{C}\right)$, an image was captured as the reference image $I_{0}$; Then, water was filled into the tank to give a depth of $20 \pm 0.1 \mathrm{~mm}$ measured by a caliper, and an image $I_{h_{0}}$ was captured when the water surface was still and flat; After that, a series of images $I(t)$ was recorded while water ripples were generated.

Coupled $I(t)$ with $I_{0}$, numerically solving the equation (2) gave $h(x, y, t)$ by the present method. On the other hand, when $I(t)$ were correlated with $I_{h_{0}}, h(x, y, t)$ from the FS-SS method can be obtained by solving the equation (3) for comparison.

The results are shown in following. In Fig. 4 (b), a snapshot of the topographic water surface from present method is shown, while the corresponding water surface reconstructed from the FS-SS method is shown in (c). The results of the two methods are in good agreement. Here the comparison is taken with removal of the spatially averaged height $\bar{h}(t)$ to emphasize the topographic surface only. The particular consideration behind this comparison is that, although the reference height $h_{0}$ is constant, $\overline{h^{\prime}}$ varies along time, which may break the fundamental assumption of the FS-SS method [1]. 200 temporal samples of $h(x, y)-\bar{h}(x, y)$, in total about 8,000,000 individual spatial points, are taken for statistics, as shown in Fig. 4 (a). Here the gray dots show the individual points, while the black squares show the bin-averaged values of the individual points, where an error bar shows the standard deviation of the individual points in a bin. Again, the good agreement between the two methods can be seen, provided that the black squares follow the black dashed line well.

The effects of initial guesses in Newton's method on the final solution $h(x, y)$ are examined. The spatially averaged depths $\bar{h}$ computed from different initial guesses $h_{0}(x, y)=15,20,25,30$ (mm) converge to the same value $\bar{h} \approx 19.93(\mathrm{~mm})$. And the topographic difference on $h(x, y)$ between the result with $h_{0}=30$ $(\mathrm{mm})$ and those with other initial guesses is only about $10^{-6}$ (mm).

In summary, the present method is independent on initial guesses. Once the initial guesses are physically meaningful, i.e. $h_{0}>0$, this method does not suffer the multi-solution issue, 

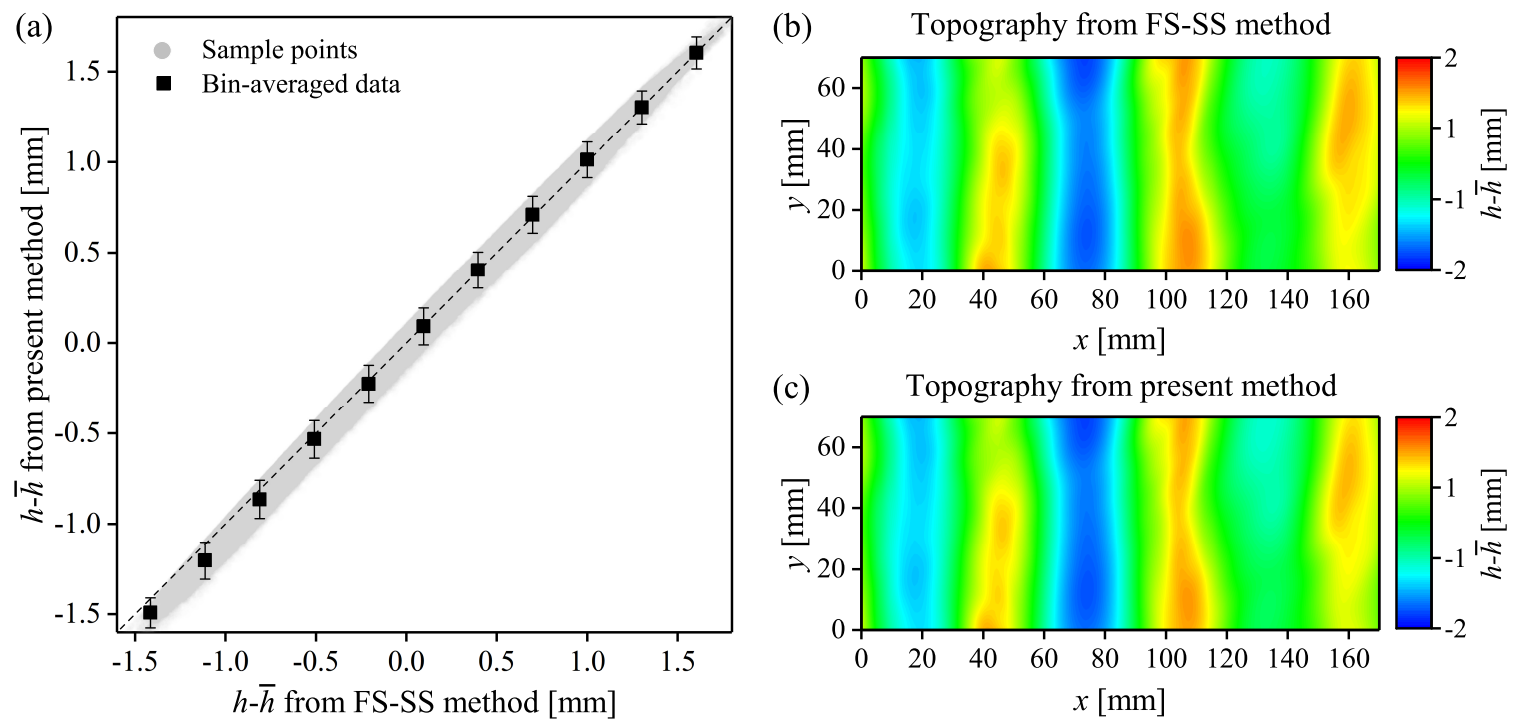

FIGURE 4. (a) THE COMPARISON OF $h-\bar{h}$ BETWEEN PRESENT METHOD AND FS-SS METHOD IN EXPERIMENTS OF DYNAMIC RIPPLES, AND AN ERROR BAR SHOWS THE STANDARD DEVIATION OF THE DATA IN THE BIN. THE COLORMAPS OF TOPOGRAPHY FROM THE FS-SS METHOD (b) AND FROM THE PRESENT METHOD (c).

which has been reported elsewhere [14]. In practice, $h_{0}(x, y)$ is suggested to be large enough for a-priori estimation of maximum depth in flow.

\section{Dam-Break Problem}

In this section, we report the experiments of a dam-break problem. Here the liquid current propagating downstream has the averaged depth changing in time.

The experiments of the dam-break problem were carried out in a lock-exchange setup as sketched in Fig. 5(a). First, water was filled into the locked portion of the tank, the left side of the gate, to the height marked by a dashed line (45 $\mathrm{mm}$ in height). When the gate was moved away vertically, the water was driven by gravity to form a dynamical current. The surface depth spatially averaged in the field-of-view of the camera changes largely in time.

The thin transparent sheet of the random dot pattern was attached to the inner bottom of the tank, while a camera was placed (approximately $1.5 \mathrm{~m}$ ) above the bottom. The experiments shared the same tank and camera-illumination system in the experiment of liquid ripples. A visualization imaging measurement was carried out at the same time to capture the height profile of the current. The visualization camera (Phantom VEO 640L) was placed (approximately $0.7 \mathrm{~m}$ ) outside the tank. A white paper attached outside the opposite wall gives an approximately uniform background. To enhance the contrast of the current to the background, the water was premixed with ink (18 $\mathrm{ml} / 1000 \mathrm{ml}$ ). The two cameras were synchronized and the sampling rate was 500 frames per second. The current height was re- constructed in the field of $110 \times 70 \mathrm{~mm}$ (in streamwise and spanwise direction, respectively). The upstream border of the measurement domain was about $390 \mathrm{~mm}$ (approximately 8.7 times the filling height) downstream the gate.

Respective examples of the reconstructed current front and current body, the flow structures in upstream, are shown in Fig. 6, where large spatial variations of the current heights can be observed. Note that the reconstructed front edges of the current front, shown in Fig. 6(a), are physically unrealistic (in line with our expectation), due to the multiple light refraction there (see Fig. 5a, and detailed discussion is given in Section LIMITATION AND DISCUSSION).

The height profile of the current body from the visualization measurement is extracted. A reference image, obtained by temporally averaging background images (without fluid), is subtracted from the visualization images. A grayscale threshold (three times of the standard deviation of the background images differing to the reference) is used to grab the height profile of the current. This profile is smoothed with a 3-point moving average operation. Given that the visualization camera has a perspective view of the flow, for comparison, $h(x, y)$ is interpolated along perspective lines (e.g., the black dash line in Fig. 5b), and the maximum height at each line is obtained to give the profile $h_{n}(x)$.

The data of the current body from two methods are compared. As shown in Fig. 7(a), $h_{n}(x)$ agrees with $h_{v}(x)$ and is bounded by the uncertainty band of $h_{v}(x)$. The individual points of $h_{n}(x)$ and $h_{v}(x)$ from about 180 temporal samples (totally 24,480 spatial points) are plotted in Fig. 7(b), where the av- 
(a)

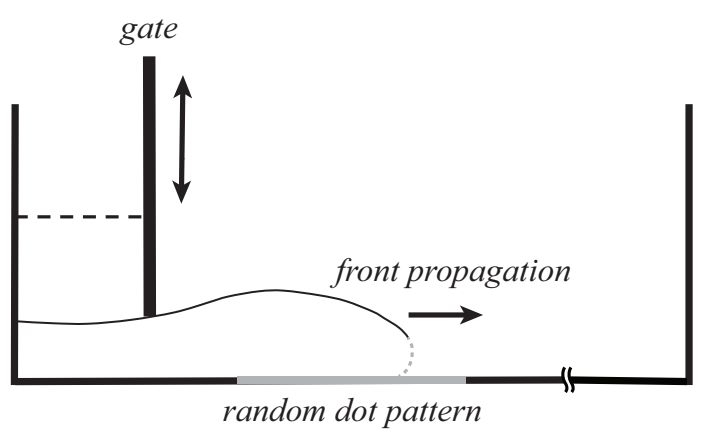

(b)

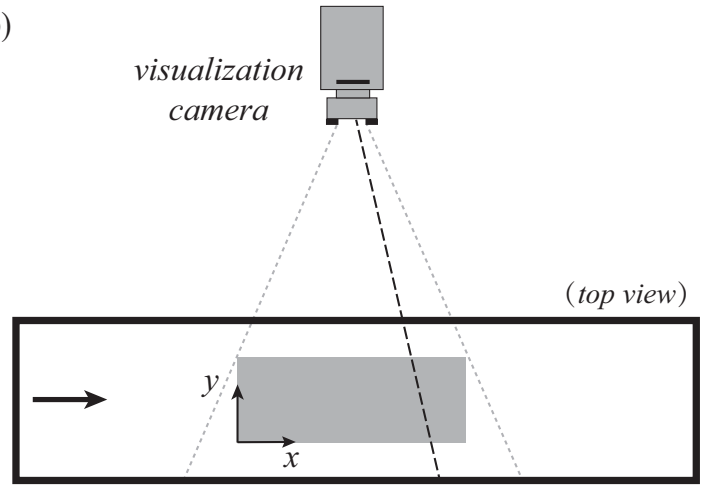

FIGURE 5. (a) SCHEMATIC OF A LOCK-EXCHANGE EXPERIMENTAL SETUP FOR A DAM-BREAK PROBLEM. THE HEIGHT OF THE LIQUID IN THE LOCKED PORTION IS MARKED BY A DASH LINE. THE LIGHT RAY IS REFLECTED MORE THAN ONCE AT VERY FRONT OF THE CURRENT (A SEGMENT OF THE DOT LINE). THE CAMERA AND ILLUMINATION SYSTEMS ARE THE SAME AS IN FIG. 3. (b) TOP-VIEW SCHEMATIC OF VISUALIZATION MEASUREMENTS. THE THICK BLACK ARROW INDICATES THE FLOW DIRECTION, AND THE GRAY RECTANGULAR MARKS THE COMPUTATION AREA OF $h(x, y)$. THE DOT LINES ENCLOSE THE FIELDOF-VIEW OF THE VISUALIZATION CAMERA, AND THE DASH LINE MARKS THE PERSPECTIVE VIEW OF THE CAMERA.
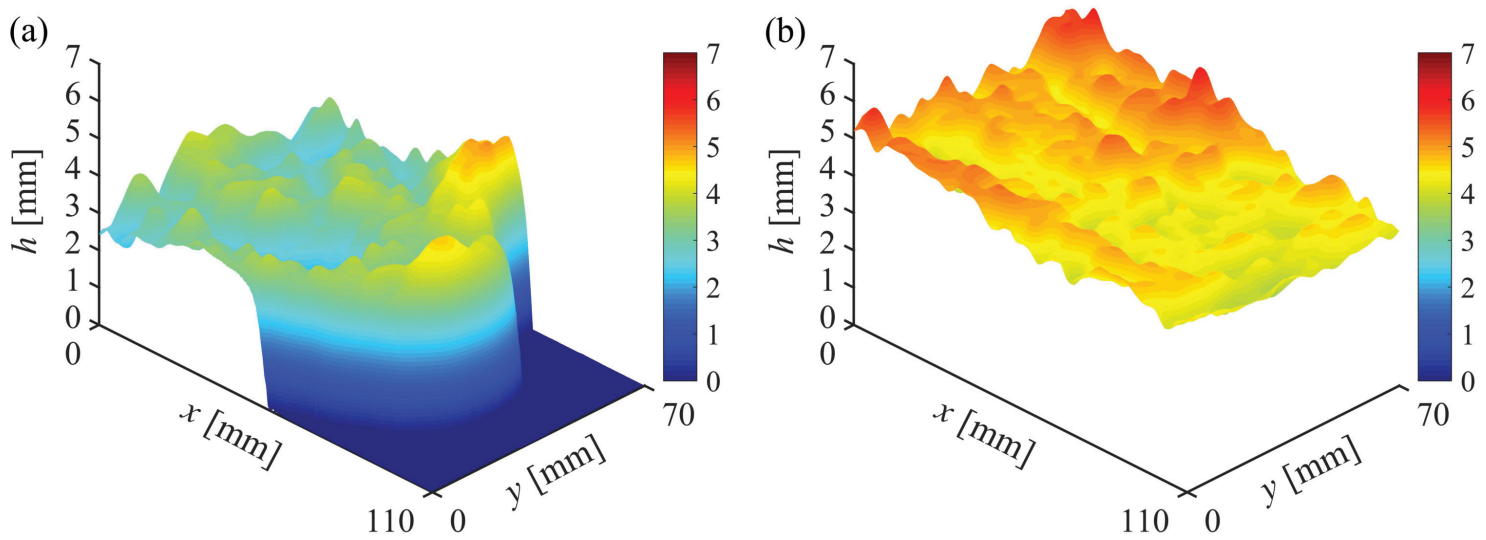

FIGURE 6. THE RECONSTRUCTED TOPOGRAPHIC PLOTS OF SAMPLES $h(x, y)$ OF THE CURRENT FRONT AND BODY: (a) CURRENT FRONT AND (b) CURRENT BODY.

eraged heights (i.e. $\sum_{i=1}^{N} h_{s}\left(x_{i}\right) / N$ ) are shown as circles. The two measurement results agree with small differences, which are attributed that the visualization measurement is affected by the inhomogeneous illumination and the two measurement domains are not exactly the same. Although a mask was used to make the visualization illumination to cover the same area of tank bottom as the computation domain of the synthetic Schlieren method, the light illumination diverges from the tank bottom to the top, which makes the visualization camera view more along the camera depth direction.

\section{LIMITATIONS AND DISCUSSION}

The surface depth $h(x, y)$ can be determined by the displacements $\delta \mathbf{r}$, when the light is refracted on the air-liquid interface once. This condition is broken when a light ray is refracted more than once or light rays cross between the pattern and the surface, i.e. formation of caustics.

As illustrated in Fig. 8(a), this condition is critical when $\boldsymbol{\beta}$ is large (border of the field-of-view). For a given cosinusoidal plane wave $h(x)=h_{p}+\eta_{0} \cos (2 \pi x / \lambda)$ with wavelength $\lambda$ and amplitude $\eta_{0}$, the critical wave amplitude is $\eta_{c}=\lambda\left[1 / 4-\beta_{x} /(2 \pi)\right]$. At the same wavelength, when $\eta_{0}>\eta_{c}$, light ray may be refracted more than once, see the dashed line in Fig. 8(a). For the snapshot in Fig. 4(c), $\lambda \approx 60(\mathrm{~mm})$ and $\beta_{x} \approx 0.08$ ( rad) give $\eta_{c} \approx 15(\mathrm{~mm})>4(\mathrm{~mm})$, and rays are refracted only once at the 

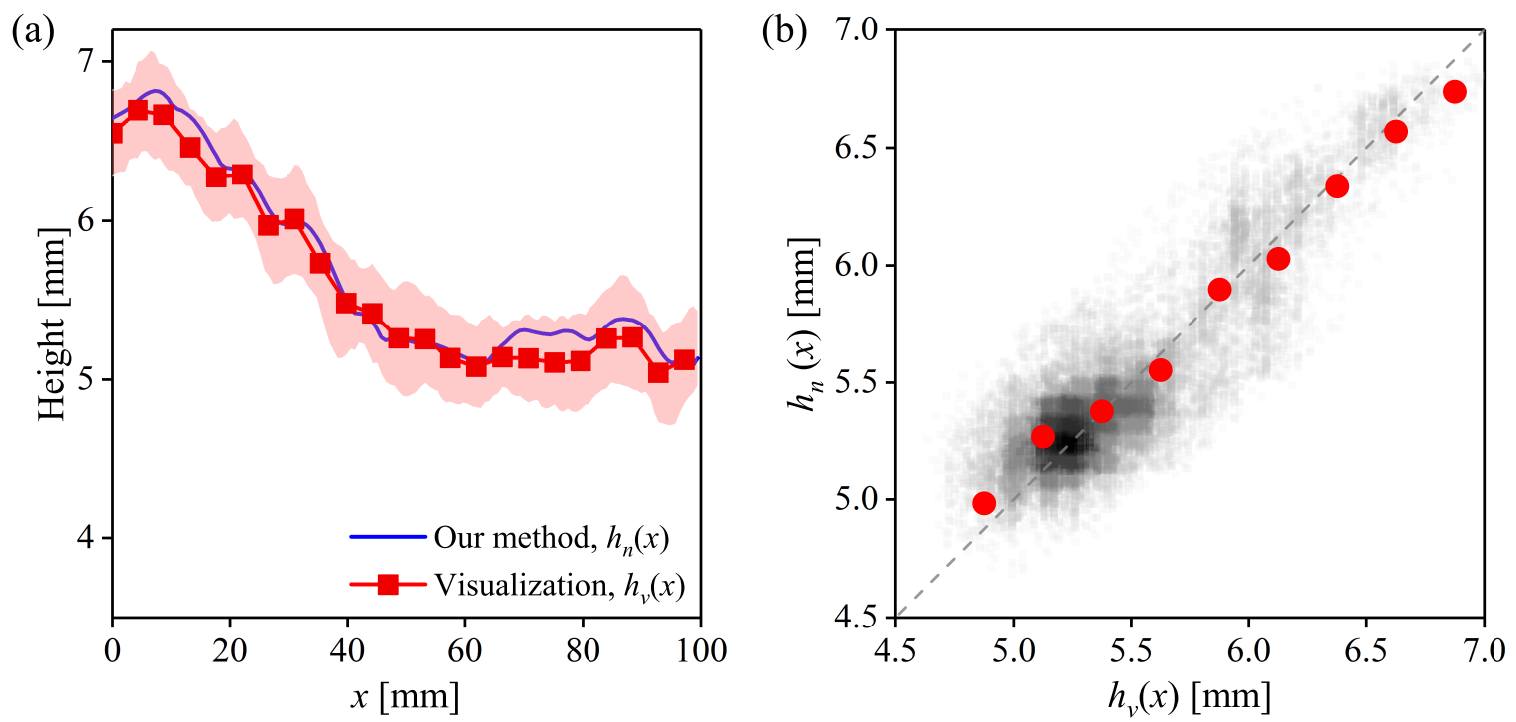

FIGURE 7. (a) THE STREAMWISE PROFILES $h_{v}(x)$ (VISUALIZATION) AND $h_{n}(x)$ (OUR METHOD) ALONG THE STREAMWISE DIRECTION $x$. RED BAND SHOW THE ERROR BAR OF VISUALIZATION MEASUREMENT AND THE SYMBOLS SHOW THE AVERAGED PROFILE. (b) $h_{n}(x)$ VERSUS $h_{v}(x)$. INDIVIDUAL SAMPLES ARE SHOWN IN POINTS AND THE GRAYSCALE LEVEL INDICATES THE PROBABILITY DENSITY FUNCTION (PDF) OF THE NUMBER OF POINTS (THE DARKER THE LARGER PDF), AND RED CIRCLES SHOW STREAMWISELY AVERAGED $h_{n}(x)$ AND $h_{v}(x)$.

(a)

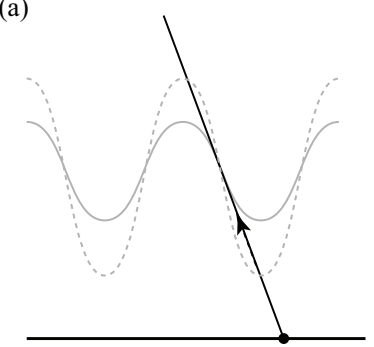

(b)

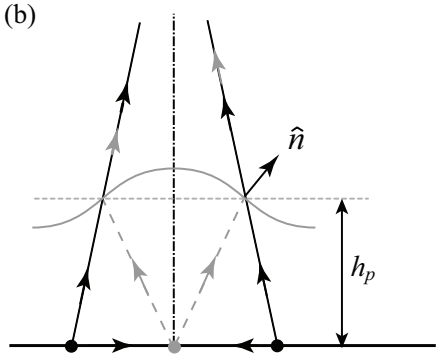

FIGURE 8. ILLUSTRATION OF BREAKDOWN CONDITIONS OF THE WORKING PRINCIPLE: (a) LARGE OSCILLATION AMPLITUDE OF SURFACE WAVE, WHERE THE LIGHT RAY IS REFRACTED MORE THAN ONCE. (b) CRITICAL CONDITION FOR FORMATION OF CAUSTICS.

surface.

The ray crossings depend on the viewing angle and the curvature of the surface [14]. Here we sketch the critical condition in Fig. 8(b): In the same cosinusoidal plane wave $h(x, y)=$ $h_{p}+\eta_{0} \cos (2 \pi x / \lambda)$, substituting into equation 2 with $\delta \mathbf{r}(\lambda / 4) \approx$ $h_{p} \beta_{x}+\lambda / 4$ and the critical depth is $h_{p, c}=\lambda^{2} /\left[8 \pi \eta_{0}\left(1-n_{a} / n_{l}\right)\right]$ taken the minimum of $h_{p}$. When $h_{p}<h_{p, c}$, no formation of caustics is expected. Moisy et al. [1] proposed a posteriori method to examine formation condition of caustics: The largest extensional strain of the displacement field needs to be bounded by 1 . This method is also applicable here. We examine all snapshots in this study and no caustics occurs. For instance, maximum eigenvalue for the snapshot in Fig. 4(c) is $0.193(<1)$ and $0.025(<0.15)$ [1].

The measurements using our method are sensitive to camera vibration, which was also suffered in FS-SS [1]. In our preliminary set-up, the camera vibration was particular evidenced and it was possibly from the cooling fan of the high-speed camera, or the vibration of the supporting profiles for the camera. Particular care is necessary to improve mounting stabilization of the camera. However, if the minimized vibration remains intolerable, measuring the vibration simultaneously with the surface depth is needed. For instance, border or corner region of a camera sensor is used to view still targets without any light refraction, while the center region of the frame is to measure the marker displacements for the surface depth. The shifts of the still targets can be then taken for evaluation of the camera vibration. Finally, the displacements from vibration needs to be removed from $\delta \mathbf{r}(x, y)$ before the reconstructed computation.

\section{CONCLUSION}

The method introduced in this study is in the category of synthetic Schlieren, or background-oriented Schlieren. This method shares similar working principle as the free-surface synthetic Schlieren (FS-SS) method. The latter relates the displacements of dots in a pattern to depth fluctuations of liquid surface around the known reference depth. Differently, the dot displacements in our method is attributed to both the surface topography 
and the mean depth, which does not need to be known. Thus, this method is applicable to the flows with temporally evolving depth. In the presented method, a calibration procedure is needed to obtain the view angle of a camera. With the known refractive indices of air and liquid (i.e. water in this study), the governing equation can be solved via the Newton's method in finitedifference scheme. The solution of the spatial distribution of the surface depth is independent on the initial guesses.

Two experiments have been conducted to examine the presented method. The experiments of water ripples, where the spatial averaged depth is nearly constant, were carried out to compare with the FS-SS method. The measurements of the two methods agree very well. In the experiments of a dam-break problem, the averaged height of the current changes with time. The results were compared with those from visualization measurements.

In use of the presented method, once the working principle is satisfied, special cares are needed on several aspects. The primary care is required on reducing camera vibration. The vibration changes the spatial relation between the camera and the target pattern, and consequently solutions are inaccurate particularly on the averaged depth of liquid surfaces. Therefore, particular care is required to improve the stability of a camera and an experimental setup. Nevertheless, when further improvement on the stability of the system is technically difficult, a camera with a lens of long focal length (for viewing the same dimension of field-of-view) is suggested that the paraxial assumption is better approximated, and we found that the solution is less sensitive to vibrations.

\section{ACKNOWLEDGMENT}

The authors thank Katja Krömer and Peter Prengel for their technical supports. Baofang Song is acknowledged for guiding our attention to numerical methods for solving the governing equation. Huixin Li thanks for the support from China Scholarship Council (No. 201804910530).

\section{REFERENCES}

[1] Moisy, F., Rabaud, M., and Salsac, K., 2009. “A synthetic Schlieren method for the measurement of the topography of a liquid interface". Experiments in Fluids, 46(6), pp. 10211036.

[2] Jähne, B., Schmidt, M., and Rocholz, R., 2005. "Combined optical slope/height measurements of short wind waves: principle and calibration". Measurement Science and Technology, 16(10), pp. 1937-1944.

[3] Aureli, F., Dazzi, S., Maranzoni, A., and Mignosa, P., 2014. "A combined colour-infrared imaging technique for measuring water surface over non-horizontal bottom". Experiments in Fluids, 55(3), p. 1701.
[4] Zappa, C. J., Banner, M. L., Schultz, H., CorradaEmmanuel, A., Wolff, L. B., and Yalcin, J., 2008. "Retrieval of short ocean wave slope using polarimetric imaging". Measurement Science and Technology, 19(5), p. 055503 .

[5] Tsubaki, R., and Fujita, I., 2005. "Stereoscopic measurement of a fluctuating free surface with discontinuities". Measurement Science and Technology, 16(10), pp. 18941902.

[6] Cobelli, P. J., Maurel, A., Pagneux, V., and Petitjeans, P., 2009. "Global measurement of water waves by Fourier transform profilometry". Experiments in Fluids, 46(6), pp. 1037-1047.

[7] Hu, H., Wang, B., Zhang, K., Lohry, W., and Zhang, S., 2015. "Quantification of transient behavior of wind-driven surface droplet/rivulet flows using a digital fringe projection technique". Journal of Visualization, 18(4), pp. 705718.

[8] Kurata, J., Grattan, K., Uchiyama, H., and Tanaka, T., 1990. "Water surface measurement in a shallow channel using the transmitted image of a grating". Review of Scientific Instruments, 61(2), pp. 736-739.

[9] Fouras, A., Jacono, D. L., Sheard, G. J., and Hourigan, K., 2008. "Measurement of instantaneous velocity and surface topography in the wake of a cylinder at low Reynolds number". Journal of Fluids and Structures, 24(8), pp. 12711277.

[10] Ng, I., Kumar, V., Sheard, G. J., Hourigan, K., and Fouras, A., 2011. "Experimental study of simultaneous measurement of velocity and surface topography: in the wake of a circular cylinder at low Reynolds number". Experiments in Fluids, 50(3), pp. 587-595.

[11] Zhang, X., and Cox, C. S., 1994. "Measuring the twodimensional structure of a wavy water surface optically: A surface gradient detector". Experiments in Fluids, 17(4), pp. 225-237.

[12] Dabiri, D., Zhang, X., and Gharib, M., 1997. "Quantitative visualization of three-dimensional free surface slopes and elevations". In Atlas of visualization, Y. Nakayama, ed. CRC Press LLC Boca Raton.

[13] Morris, N. J. W., 2004. Image-based water surface reconstruction with refractive stereo. University of Toronto.

[14] Gomit, G., Chatellier, L., Calluaud, D., and David, L., 2013. "Free surface measurement by stereo-refraction". Experiments in Fluids, 54(6), p. 1540.

[15] Raffel, M., 2015. "Background-oriented Schlieren (BOS) techniques". Experiments in Fluids, 56(3), p. 60.

[16] Charruault, F., Greidanus, A., Breugem, W.-P., and Westerweel, J., 2018. "A dot tracking algorithm to measure free surface deformations". In Proceedings 18th International Symposium on Flow Visualization, ETH Zurich.

[17] Moisy, F., 2018. PIVMat 4.10: A PIV Post- 
processing and data analysis toolbox. https:

/ / www . mathworks.com/matlabcentral/

fileexchange/10902-pivmat-4-10.

[18] Raffel, M., Willert, C. E., Scarano, F., Kähler, C. J., Wereley, S. T., and Kompenhans, J., 2018. Particle image velocimetry: a practical guide. Springer. 